\title{
Fibre optic sensing system for monitoring of current collectors and overhead contact lines of railways
}

\author{
Kerstin Schröder ${ }^{1}$, Manfred Rothhardt ${ }^{1}$, Wolfgang Ecke ${ }^{1}$, Uwe Richter ${ }^{2}$, André Sonntag ${ }^{2}$, and \\ Hartmut Bartelt ${ }^{1}$ \\ ${ }^{1}$ Leibniz Institute of Photonic Technology, Albert-Einstein-Str. 9, 07745 Jena, Germany \\ ${ }^{2}$ Eurailscout Inspection \& Analysis b.v., Torellstr. 1, 10243 Berlin, Germany \\ Correspondence to: Kerstin Schröder (kerstin.schroeder@leibniz-ipht.de)
}

Received: 19 September 2016 - Revised: 16 December 2016 - Accepted: 26 December 2016 - Published: 6 February 2017

\begin{abstract}
Fibre optic sensors are excellent tools to use for monitoring high-voltage current collectors. Because of their small cross section and electrical neutrality, they are easily integrated into the current collector strip and are well specialized for detection of high-speed load events. The conventional contact force measurement with four force sensors below the collector strips can also be simplified by using fibre optic force and acceleration sensors.
\end{abstract}

\section{Introduction}

The deregulation of the European Union (EU) railway market and the necessary interoperability of railways and tracks has increased the need for easy-to-use online monitoring of railway tracks and catenaries (in the best case available daily). Great efforts have been put into the development of overhead contact line (OCL) monitoring systems. Permanent monitoring of the load conditions allows the prediction of the resulting wear, and helps to optimize service and repair cycles on the OCL.

Some measurement techniques for OCL monitoring, such as contact force measurements between pantographs and the OCL, are already well established. Boundary conditions are defined in a European standard (EN 50317). Other techniques, for instance the detection of disturbances (misalignments or intruders), are still under development or under optimization.

For heavy load trains or steep tracks, some trains are driven with two locomotives and two pantographs. Because of strong dynamics of the catenary, this can often lead to contact loss of the second pantograph. In such cases, measurement and control of the contact force is of high interest.

Measurements at the interface between OCLs and current collectors with electrical sensors are complex and prone to interference from the high electrical voltages and the strong changes in electric and magnetic fields. In contrast, fibre op- tic sensors have the potential of easy access to such measurements due to their small size, immunity to electromagnetic fields, and their intrinsic electrical insulation. For this reason, research is currently underway to utilize fibre optic sensors for monitoring purposes in current collectors and OCLs (Schröder et al., 2012; Theune et al., 2004; Boffi et al., 2009).

Maladjusted catenary elements or intruders into the catenary region cause hits on the current collectors. They can damage the carbon of the collector strip and cause serious traffic incidents. In Fig. 1, two used collector strips are shown with their typical stress marks. At the edges, smaller or bigger parts of the carbon have been chipped away. The lower photograph shows a seriously damaged collector strip. Such a strip can seriously damage the OCL.

The aim of this sensing system is the detection of low-level hits already below the damage threshold, in order to identify sources of damages in a very early state and to provide timely maintenance on demand.

Fibre Bragg grating (FBG) optical fibre sensors and especially draw tower FBG (Chojetzki et al., 2005) are the preferred kinds of sensors for monitoring purposes on this interface (Schröder et al., 2013; Comolli et al., 2010). This is due to their ability to have many sensor elements multiplexed along a single optical fibre, and to the fact that the quantitative load result is derived from a spectral shift, which is immune to light intensity variations. Currently, such investigations are focused on the optimization of the sen- 


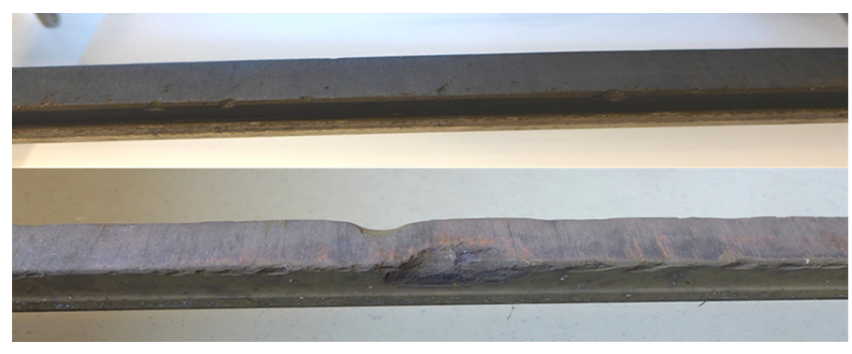

Figure 1. Top: used collector strip with clear abrasion marks on the edges. Bottom: used collector strip with serious damage.

sors and their instrumentation. In this paper, results are reported from the investigation of two different measuring techniques: quantitative logging of (i) fast hit events and (ii) the stationary acceleration-compensated contact force measurement (Fig. 2).

\section{FBG sensor interrogating system}

There are different ways known to interrogate load results from FBG sensors. The sensor system used in these studies is based on a broadband light source and a line-based compact spectrometer with a photo-detector line, comprising depolarization components, fibre optic connectors to four sensor lines, fast- and low-noise digital signal processor (DSP) electronics, Ethernet data communication, and a break-free power supply (Fig. 3).

All these components are placed in a box with dimensions of $18 \mathrm{~cm} \times 28 \mathrm{~cm} \times 10 \mathrm{~cm}$, allowing easy integration into a locomotive. The interrogation unit can simultaneously read 32 FBG sensors at 5000 measurements per second, with a strain resolution of about $1 \mu \mathrm{m} \mathrm{m}^{-1}$ per sensor. In order to obtain the position of measured events along the rail track, the position data of the locomotives are fed directly into the interrogator's DSP and recorded simultaneously with the load data. For stabilization of long-term contact force measurements, two fibre optic depolarizers (Lyot type at the light source and a spatial mode mixer in front of the spectrometer, respectively) minimize the influences of FBG birefringence and polarization dependencies of the spectrometer. The temperature dependence of the spectrometer was measured in a calibration procedure. So, no measurement drifts occur and the strain measurement errors are within $\pm 1 \mu \mathrm{m} \mathrm{m}^{-1}$. Each sensor head, which is mounted below the collector strip, comprises an FBG force sensor and an FBG acceleration sensor, which are integrated along a single optical fibre.

\section{Integrated collector strip sensors}

Because of their convenient small dimensions, the integration of fibre optic sensors, and especially of fibre Bragg gratings, is easily achieved inside the current collector. In this case, the sensors are very close to the interacting interface between the

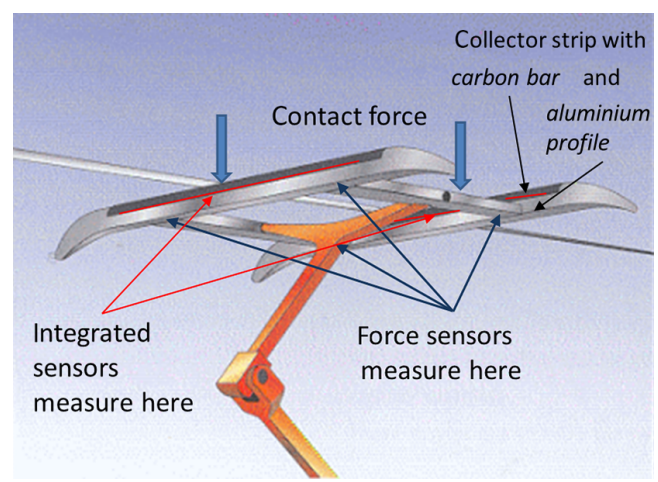

Figure 2. Overhead contact line in contact with a pantograph head containing two collector strips with integrated sensors. Additional force sensors are included below the collector strips.

collector strip and the overhead contact line. The closer the sensors are to the interface, the more accurately the monitoring of fast hits can be performed, because signal delays and mechanical damping are minimized. An integration of the sensor fibre into the interface between the carbon bar and the aluminium carrier before their assembly showed the best sensing performance and safety for the sensors.

The collector strip with integrated FBG sensors (shown schematically in Fig. 4) has provided outstanding application results for the detection and measurement of hard hits on the carbon bar in the driving (horizontal) direction.

\subsection{Hit characteristics}

Experiments showed two principal types of hit events: "soft hits" and "hard hits".

"Soft hits" can be caused by short-term locomotive accelerations, e.g. from rail steps or vibration of the pantograph on the catenary. Typical frequencies are 220 to $250 \mathrm{~Hz}$ with corresponding slow peak rise times of $\sim 1 \mathrm{~ms}$ and low damping. These "soft hits" can also be measured with acceleration sensors below the collector strips.

"Hard hits" are characterized by short rise times (50 to $200 \mu \mathrm{s}$ ) and strong attenuation (examples are given in Figs. 5 and 6). They can cause fast and strong reactions on the carbon bar and on the embedded strain sensors, which can only be detected with a correspondingly fast measurement system and only in close vicinity to the hit point. "Hard hits" usually occur when the carbon bar is hit by OCL steps and kinks, or by intruders. They are dangerous for the carbon material and should be avoided.

The example in Fig. 5 was measured under laboratory conditions: a hit on the carbon bar was executed with a metal test pendulum. The hard hit on the carbon excited transversal and longitudinal surface waves, which spread at velocities of 3.3 and $5.7 \times 10^{3} \mathrm{~m} \mathrm{~s}^{-1}$, respectively, along the carbon bar, and were reflected at its free ends. 


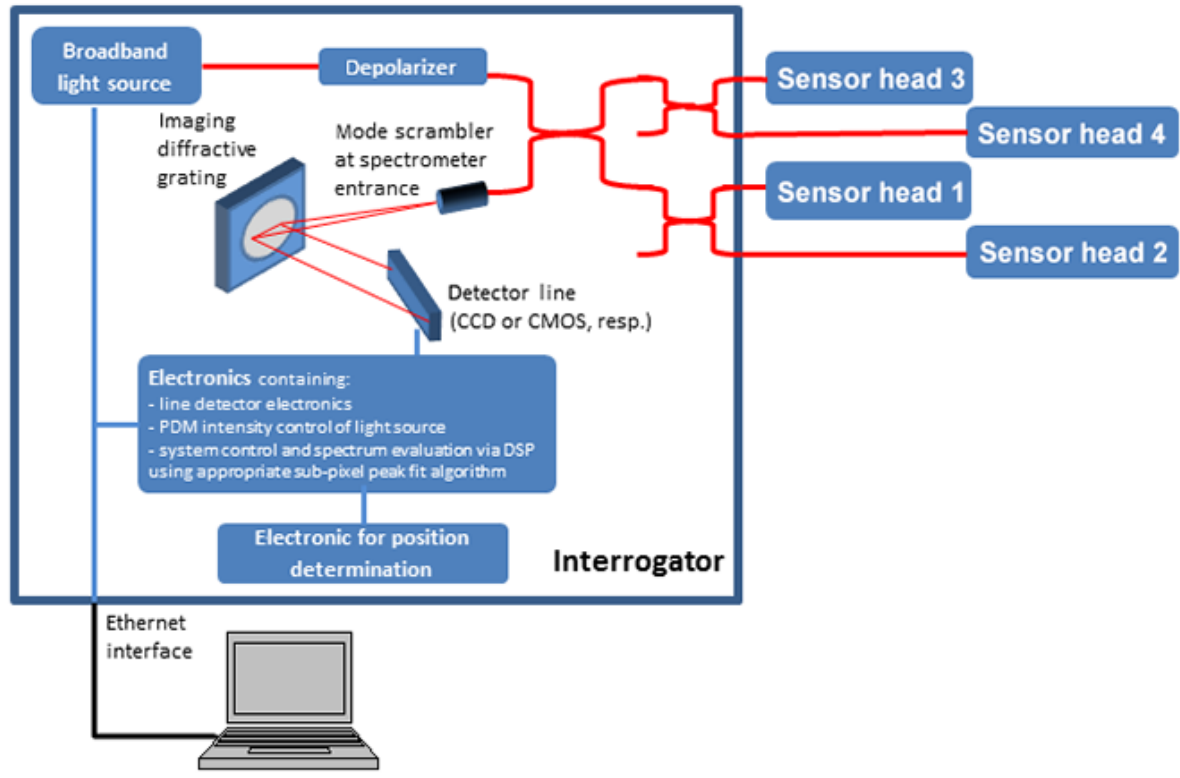

Figure 3. The FBG sensor interrogating system contains a broadband light source (superluminescent diode), which is depolarized with an appropriate fibre optic depolarizer. The reflected signals from the FBGs in the four sensor heads are spectrally analysed in a compact spectrometer containing a mode scrambler for optimal entrance slit illumination, an imagine diffractive grating, and a line detector (chargecoupled device - CCD - for the "slow" version, CMOS for the interrogation speed of up to $5 \mathrm{kHz}$ ). The heart of the interrogator electronics is a digital signal processor (DSP), which controls the intensity of the light source via pulse duration modulation (PDM), corrects the temperature dependence, evaluates the peak spectra using appropriate sub-pixel approximation, integrates the position data, and sends the sensor data via an Ethernet interface to a displaying and saving device.

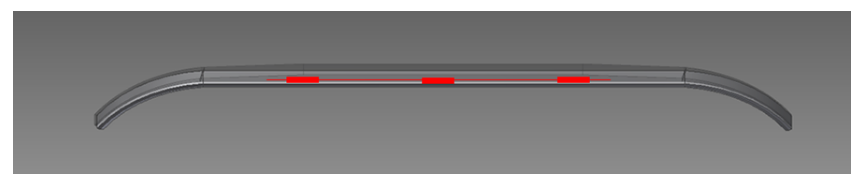

Figure 4. Collector strip with schematic representation of integrated fibre optical sensors (red). They are positioned on the boundary between the aluminium profile and the carbon bar, close to the edge, and were used successfully to detect hits in a horizontal (driving) direction.

These "hard hits" can usually not be detected by sensors far from the interface, below the collector strips, but only with integrated sensors, because of the strong signal attenuation. The sensor system must interrogate at data rates of at least 5000 measurements $\mathrm{s}^{-1}$ to be able to identify such hits. More detailed laboratory investigations of hit responses have been performed with 20000 measurements $\mathrm{s}^{-1}$.

From such laboratory tests, the dependencies between signal amplitude and hit energy have been found to be linear for these collector strips, with a slope of $74 \mu \mathrm{m} \mathrm{m}^{-1} \mathrm{~J}^{-1}$ (Schröder et al., 2013). This sensitivity depends slightly on the hit position along the collector strip. Since position detection was not yet integrated into this specific system, a slight systematic error occurs for the energy estimation, such as in the example of the test run shown in Fig. 6.

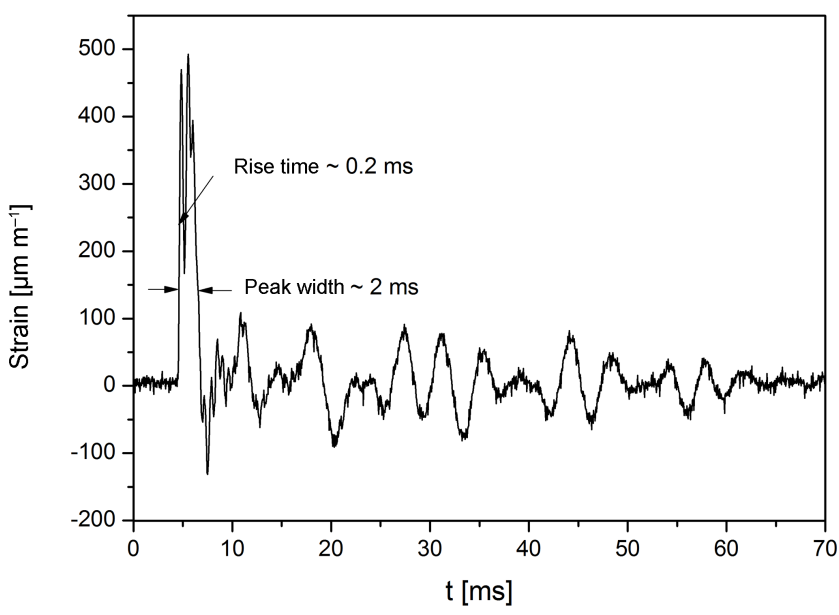

Figure 5. Sensor response from a hit with a metal pendulum on the carbon bar ("hard hit").

For an evaluation of hit measurements, two factors were identified as most important. The first one is the hit type ("soft" or "hard" hit); the second one is the hit intensity. Figure 7 shows a part of a track measurement with an extraordinarily high density of "events" and a variety of intensities. Red arrows mark the events, which were found by their characteristics to be hard hits. Hard hits can occur with high and 


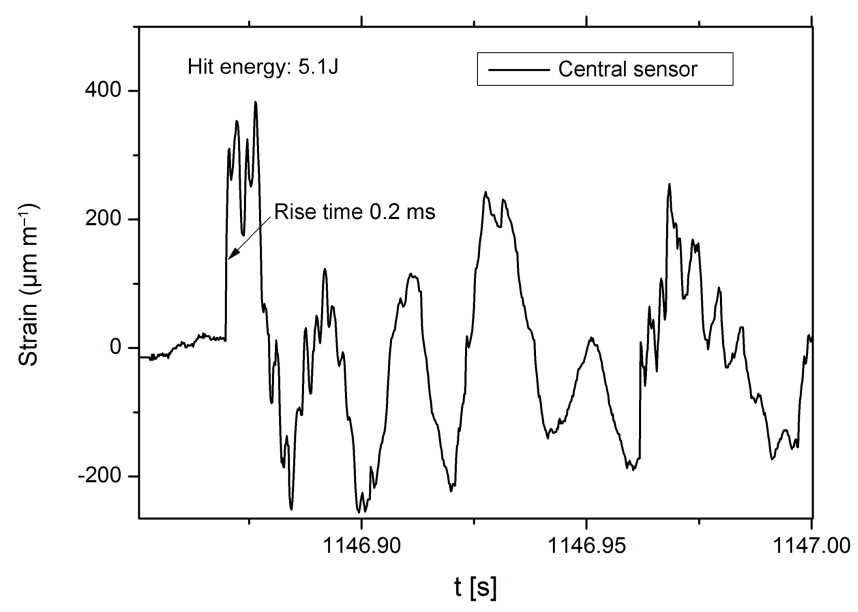

Figure 6. Signal detected during a test run. Passing a section insulator at $37 \mathrm{~km} \mathrm{~h}^{-1}$ caused this hit. The short rise time is typical for a "hard hit". The energy of the hit was estimated to be $5.1 \mathrm{~J}$.

low intensities. To classify these hits, an injury level had to be defined. It should be set below but close to the destruction level. A general definition of a destruction level only by a measured hit energy is not possible, because other factors, such as hit area (close to the edge, hit with thin tip) and the history and quality of the carbon material, as well as the construction of the fixation of the collector strip within the pantograph, play important roles. In laboratory experiments with new collector strips hit with $50 \mathrm{~J}$ at the same point with a large-area mass, most strips crack after the third hit. But when a mass with a spherical tip is used, hit energies of $20 \mathrm{~J}$ can chip away parts of the carbon. Therefore the highest alarm level was set at a hit energy of $20 \mathrm{~J}$. For the collector strips under investigation, this would correspond to a strain maximum of about $1500 \mu \mathrm{m} \mathrm{m}^{-1}$. All events with a level below $200 \mu \mathrm{m} \mathrm{m}^{-1}$ are qualified as harmless. The detection limits of the measurement system are at a maximum of $4000 \mu \mathrm{m} \mathrm{m}^{-1}$ and a minimum of $2 \mu \mathrm{m} \mathrm{m}^{-1}$, far above and below these injury levels, which allows us to fulfil this measurement task easily.

\section{Contact force measurement}

A first concept sought to use the collector strip as a bending beam in order to transform stationary contact forces into strain, which can then be measured. However, an issue arose due to the low thermal conductivity of the upper strip material (carbon) and the different thermal elongation compared to the aluminium carrier sheet. This led to local heating by sunlight or a high electrical current, causing deformation of the strip, which was detected much earlier by the strain sensors compared to the true temperature change. The resulting errors are discussed in detail in Schröder et al. (2012).

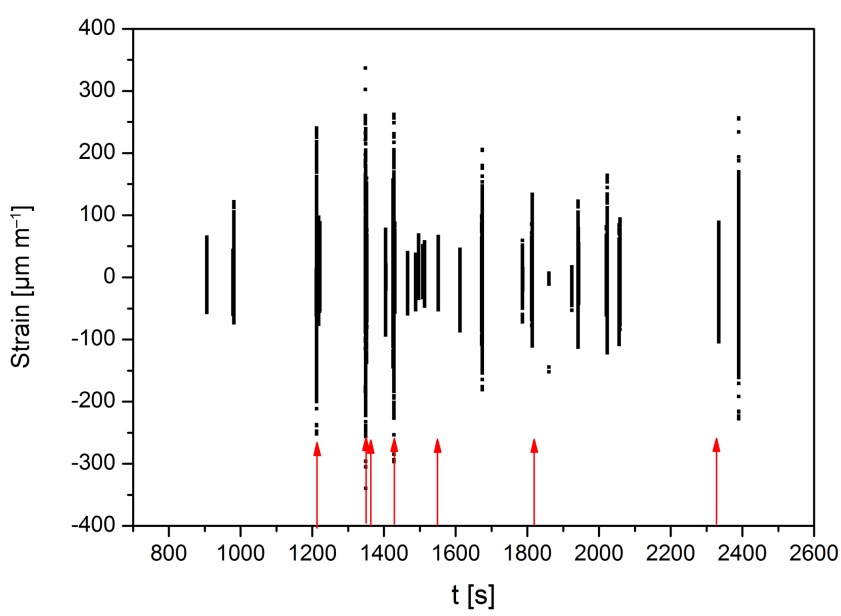

Figure 7. Part of a track measurement with an extraordinarily high density of events in a track region with many switches. The measured strain data of identified events are given as point plots in black. Red arrows mark the events from "hard" hits. "Hard" hits can occur with high and low intensities. Hits under the level of $200 \mu \mathrm{m} \mathrm{m}^{-1}$ were identified as harmless, hits below the level of $1500 \mu \mathrm{m} \mathrm{m}^{-1}$ as very serious.

As a consequence, the contact force measurement is now done with additional fibre optic force sensors fixed below the collector strips.

The contact force $F_{\mathrm{C}}$ between the contact line and the collector strip is the sum $F_{\mathrm{S}}$ of the forces $F_{i}$ measured below the collector strips corrected by the inertial forces $F_{\text {in }}$ on the positions of the force measurement and the aerodynamic force $F_{\text {aer }}$ (Eq. 1) (Kießling et al., 2011).

$F_{\mathrm{C}}=F_{\mathrm{S}}+\mathrm{F}_{\text {in }}+F_{\text {aer }}$

The aerodynamic force $F_{\text {aer }}$ is dependent mainly on the pantograph, the collector strip geometry and the driving speed. The speed dependence needs to be measured in a calibration test run for each new set. With these calibration data, only the speed of the locomotive needs to be known for the correction of aerodynamic forces. Therefore the contact force can be described as

$F_{\mathrm{C}}=\sum_{i=1}^{4} F_{i}+\sum_{i=1}^{4} F_{\mathrm{in}_{i}}+F_{\mathrm{aer}}(v)$.

The inertial forces are calculated by

$F_{\text {in }}=m_{\text {above }} \cdot \sum_{i=1}^{4} a_{i}$,

where $m_{\mathrm{above}}$ is the whole mass above the sensors and $a_{i}$ are the accelerations at the measurement points $i$.

To minimize the measurement uncertainty for $F_{\mathrm{C}}$ by inertial forces, the force and acceleration sensors should be mounted as close as possible below the collector strips. The mass acting above the sensors (in this case mainly the collector strips) should be as low as possible. 


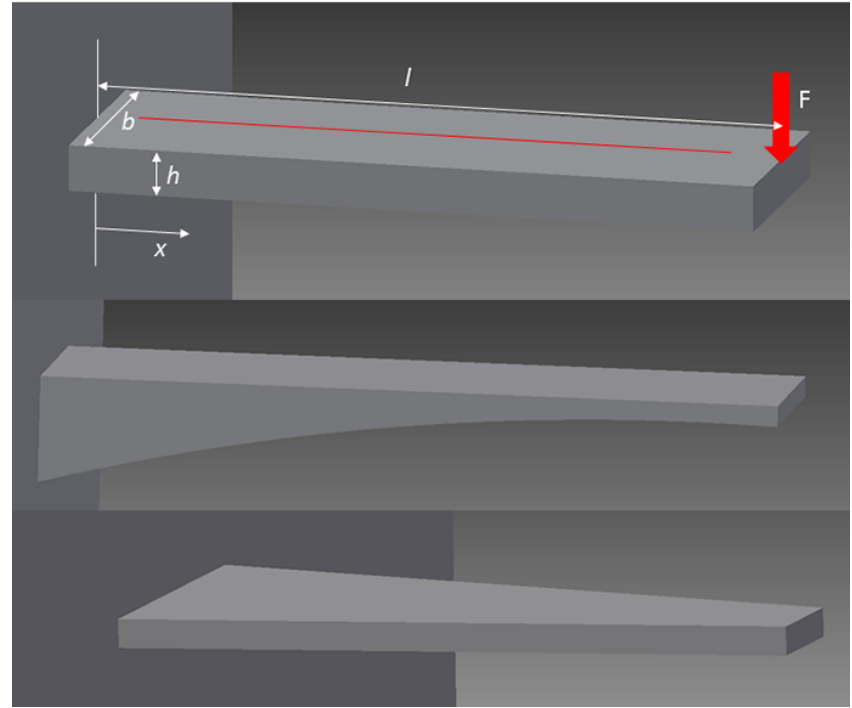

Figure 8. (a) Homogeneous bending beam with definitions of parameters. (b) Bending beam with varying thickness $h$. (c) Bending beam with varying width $b$.

\subsection{Contact force sensors}

The contact force sensors are made as S-type force sensors (scheme in Fig. 10a, photograph in Fig. 9a).

It is important to understand that FBG sensors, compared to electrical strain gauges, show different properties when applied across an inhomogeneous strain distribution. Electrical strain gauges usually integrate the strain distribution over the measuring area and show a "medium strain" when they see inhomogeneous strain distribution. In contrast, FBG sensors show a deformation of the Bragg spectrum, which can lead, depending on the kind of inhomogeneity and the interrogation algorithm, to significant measurement errors. In order to avoid a Bragg peak deformation under load, the transducer has been designed to have homogeneous surface strain in the region of the FBG under all load conditions. For a vertical point force at distance $l$ on a bending beam of constant Young's modulus $E$, of width $b$, and height $h$ (definitions in Fig. 8a), the strain $\varepsilon(x)$ on the red line on the surface, where the sensor will be placed, depends on $x$ according to

$\varepsilon(x)=\frac{6 \cdot F \cdot(l-x)}{E \cdot b(x) \cdot(h(x))^{2}}$.

In order to obtain a strain $\varepsilon$ independent of $x$, i.e. $\varepsilon(x)=\varepsilon_{0}$, the width $b$ and the height $h$ of the bending beam can be varied independently.

Keeping the width constant, $b(x)=b_{0}$, the height $h$ has to follow the condition

$h(x)=h_{0} \cdot \sqrt{1-\frac{x}{l}}$

with $h_{0}$ the height at the beam root $x=0$ (Fig. 8b).
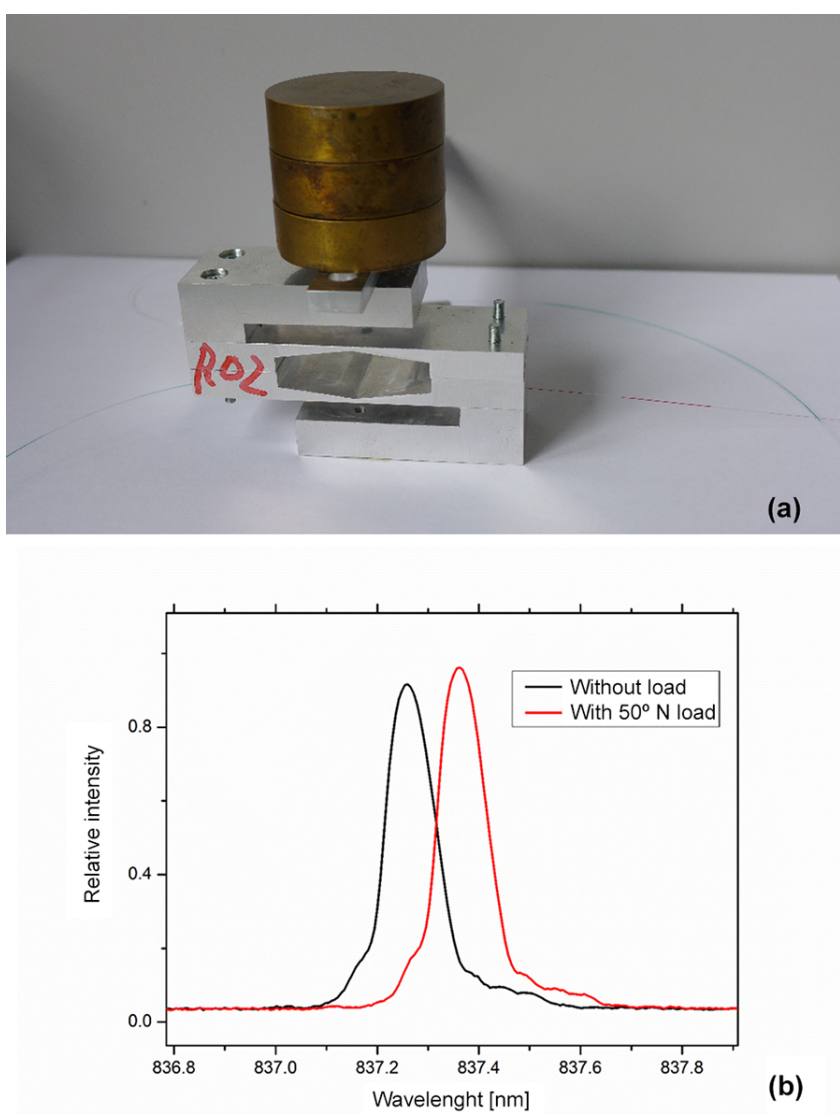

(b)

Figure 9. (a) S-type difference sensor R02 with bending beams of varying thickness for force detection (with test masses as load). (b) The shape of the Bragg peak of sensors applied to sample R02 is not significantly changed if the sensor is loaded with $50 \mathrm{~N}$.

Keeping the height constant $h(x)=h_{0}$, the width $b$ has to follow the condition

$b(x)=b_{0} \cdot\left(1-\frac{x}{l}\right)$

with $b_{0}$ the width at the beam root $x=0$ (Fig. $8 \mathrm{c}$ )

A robust construction with constant beam width was prepared by water jet cutting from an aluminium block (Fig. 9a). Fig. $9 \mathrm{~b}$ shows that there is no deformation of the sensor spectra under load.

With this solution, the variety of materials is very limited because many high-strength materials are available mainly as panel sheets and get their strength from special surface conditioning. Using bending beams from panel sheets, one needs to work with a constant beam height and linear decrease in the width (similar to Fig. 8c). All the following examples work with such constructions.

To achieve a high sensitivity with good reproducibility, it is necessary to choose a mechanical transducer material with a very high elasticity limit $\mathrm{Rp} 0.2$ (proof stress for maximum $0.2 \%$ strain). We tested spring brass as well as steel-type $\mathrm{Ni}$ - 
vaFlex 45/18 with an Rp0.2 of 500 and $1800 \mathrm{MPa}$, respectively.

A sensitivity of $14.9 \mu \mathrm{m} \mathrm{m}^{-1} \mathrm{~N}^{-1}$ was achieved for the spring brass. With the steel NivaFlex $45 / 18$, the sheet can be chosen to be much thinner while maintaining the same strength. With the thinner sheet, a higher sensitivity can be achieved. For all the following examples we worked with sensor samples from NivaFlex $45 / 18$, which had a sensitivity of $22 \mu \mathrm{m} \mathrm{m}^{-1} \mathrm{~N}^{-1}$. With a safety factor of 1.8 for the elastic deformation region, this allows a measurement range of up to $200 \mathrm{~N}$. Using an interrogator with an accuracy within $\pm 0.5 \mu \mathrm{m} \mathrm{m}^{-1}$, a measurement accuracy of $\pm 23 \mathrm{mN}$ can be achieved.

In general, the readout of strain sensors is cross-sensitive to temperature, and thus a specific concept is necessary for its compensation. As mentioned above, an anti-symmetric sensor with equal strain responses but opposite sign of the two single strain sensors $\varepsilon_{\mathrm{p}}$ and $\varepsilon_{\mathrm{n}}$ (Fig. 10a) is used in this concept. The force is calculated from the difference in the readouts of both sensors, while temperature is calculated from their sum.

Temperature tests of four sensor heads, consisting of two double FBGs per sensor head, in an oven with temperatures between 20 and $45^{\circ} \mathrm{C}$, were performed in order to check this concept. The temperature reading from the sensors has an accuracy within $\pm 0.5^{\circ} \mathrm{C}$ (Fig. 10b). The measured force reading change caused by temperature changes is within $\pm 0.5 \mathrm{~N}$ (Fig. 10c).

\subsection{Acceleration sensors}

In order to compensate for the measured force values for inertial forces, acceleration sensors are added to the contact force sensor heads.

Different types of FBG-based acceleration sensors can be found in the literature. In Antunes et al. (2011) and Jiang and Yang (2013) an FBG is stretched by a moving inertial mass. In Mita et al. (2001) and Au et al. (2008) a bending beam - or rather a cantilever-based FBG accelerometer - is investigated.

In our work, we developed two different types of cantilever-based FBG accelerometers. Both of them are based on bending beams, which are bent by the reaction of an inertial mass to acceleration. The bending beam has triangular width in order to generate a constant strain distribution (Eq. 6, Fig. 8c). Prototype no. 1 used a spring steel-bending beam with an FBG mounted $2 \mathrm{~mm}$ above the surface and a mass of $60 \mathrm{~g}$ fixed on the tip of the triangular bending beam.

For the second prototype (no. 2), the mass was fixed by steel springs, and it drives an additional steel triangle with an attached FBG (see Fig. 11c). In this configuration, the mass holder springs and the sensor-bending beam can be optimized separately.

Both types were tested for their sensitivities and resonance frequencies with the help of a shaker (TIRA S 51128). For
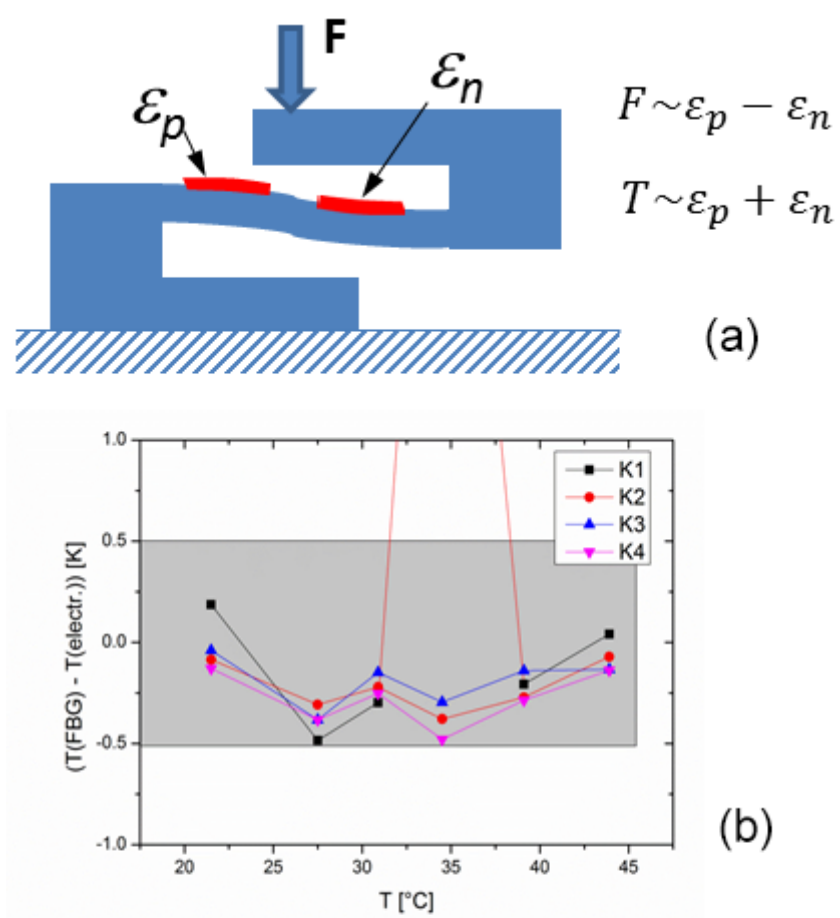

(b)

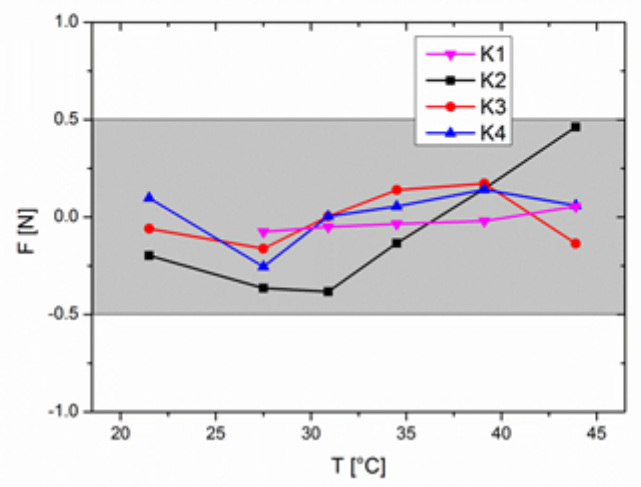

(c)

Figure 10. (a) Concept for temperature compensation: the sensor was designed in a way that a vertical force will induce a positive strain on sensor $\varepsilon_{\mathrm{p}}$ and a negative strain with the same magnitude on sensor $\varepsilon_{\mathrm{n}}$. Temperature changes induce for $\varepsilon_{\mathrm{p}}$ and $\varepsilon_{\mathrm{n}}$ readings with the same magnitude and the same sign. So, the difference $\varepsilon_{n}-$ $\varepsilon_{\mathrm{p}}$ is independent of temperature but dependent on the applied force. (b) Accuracy of temperature measurements with four sensor heads in an oven at temperatures between 20 and $45^{\circ} \mathrm{C}$, in comparison to electrical temperature sensors. (c) The force readings of all sensor heads are temperature-independent.

prototype no. 1 , a sensitivity of $36 \mu \mathrm{m} \mathrm{m}^{-1} \mathrm{G}^{-1}$ (Fig. 11a) and a resonance frequency of $192 \mathrm{~Hz}$ (Fig. 11b) were measured. For prototype no. 2, the sensitivity was strongly dependent on the fine adjustment. Sensitivities were measured in the range of 24 to $72 \mu \mathrm{m} \mathrm{m}^{-1} \mathrm{G}^{-1}$. The lowest resonance was weak, but at a very low frequency of approximately $86 \mathrm{~Hz}$.

To check the performance of the acceleration sensor and to test the compensation of the inertial force of the force sensor, 

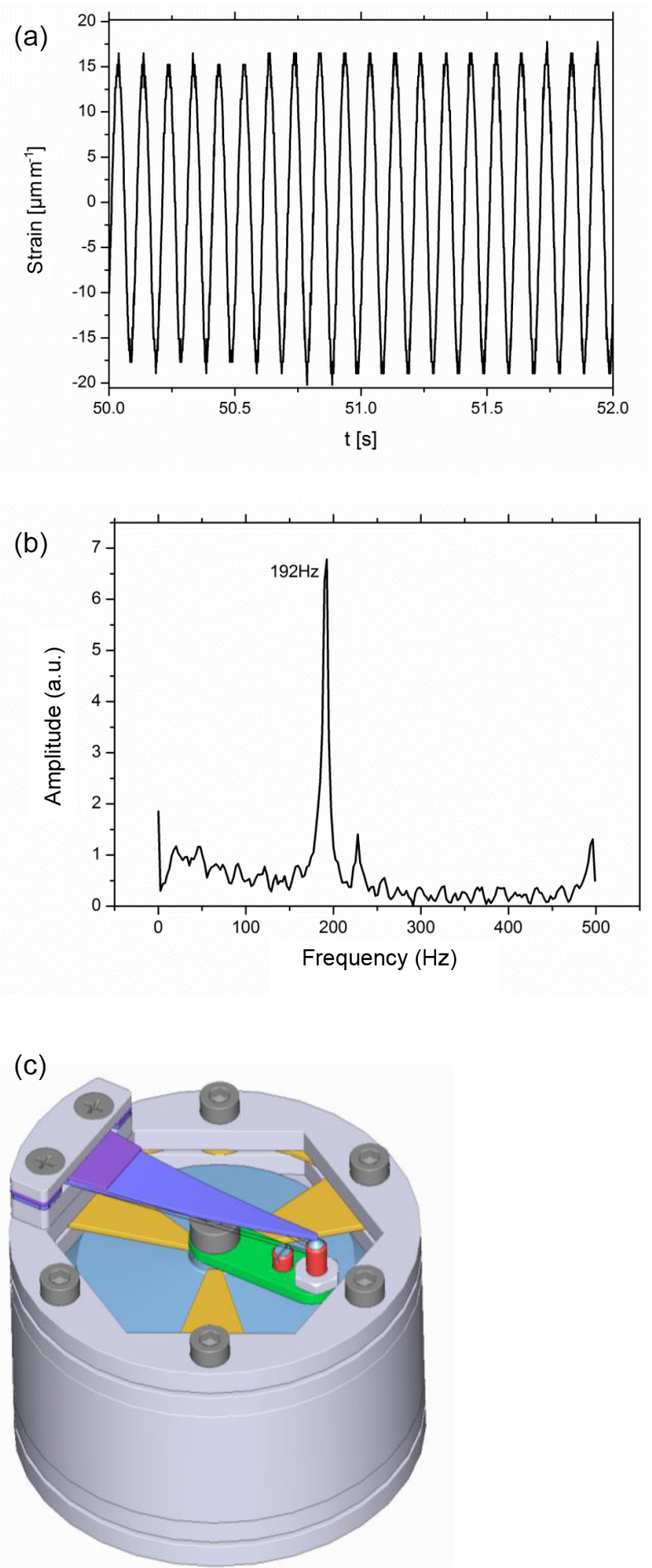

Figure 11. (a) Reaction of sensor prototype no. 1 to a sinusoidal excitation of frequency $10 \mathrm{~Hz}$ and amplitude $2.5 \mathrm{~mm}$. (b) The resonance frequency of sensor prototype no. 1 is $192 \mathrm{~Hz}$. (c) Design of sensor prototype no. 2. The mass (blue) of about $50 \mathrm{~g}$ was fixed with steel springs (yellow) and it moved the triangular bending beam (violet) on top with the attached FBG sensor (not shown here).

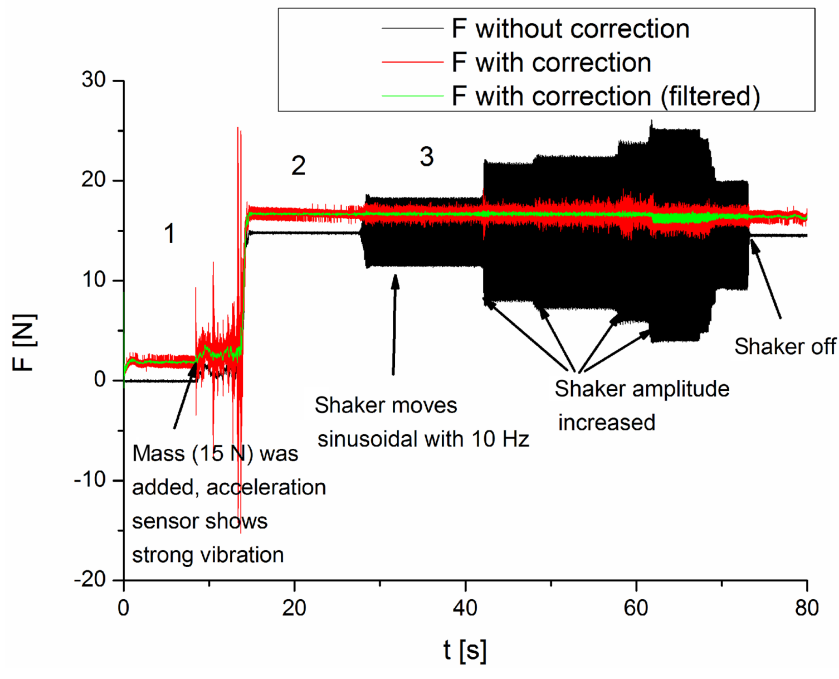

Figure 12. Contact force sensor with an acceleration sensor in a test on a shaker. Detailed descriptions of the experiments are given in the text. Black: contact force results without acceleration compensation (very low noise during standstill). Red: contact force results with acceleration compensation $\left(\sim 35 \mu \mathrm{m} \mathrm{m}^{-1} \mathrm{G}^{-1}\right.$ and $\left.192 \mathrm{~Hz}\right)$. An offset was added in the graphic. Green: contact force results in red, with a $20 \mathrm{~Hz}$ low-pass filter (corresponding to EN50317).

both types of sensors were vibrated together in the shaker. The force sensor was loaded with a mass exciting a gravity force of $15 \mathrm{~N}$. Results of a sample measurement are given in Fig. 12. In segment 1, the system is undisturbed initially, but fixing the additional mass excites a strong high-frequency disturbance. This cannot be compensated for by the acceleration sensor (red curve). However, it is eliminated after application of a $20 \mathrm{~Hz}$ low-pass filter (green curve), as it is mandatory in European standard EN50317. In segment 2, the mass is applied and the sensor is not moved. In segment 3, the shaker moved the sensor by a sinusoidal waveform pattern at a frequency of $10 \mathrm{~Hz}$. The red curve (contact force with compensation of inertial force) is expected to be smooth in this section, and these expectations are fulfilled very well. After the mandatory filtering, a residual deviation from the $15 \mathrm{~N}$ force set value was kept within $\pm 0.5 \mathrm{~N}$.

\subsection{Sensor integration}

The interface between the force sensor and the collector strip was developed by a third party. It fulfils the task of negligible low cross-sensitivities to further forces on the collector strip. The task of reproducible transition of the contact force from the collector strip to the sensor has not yet been solved by this technique; thus, a revision is under investigation.

\subsection{First test drive}

A first test drive took place on a track of the lignite-fired power plant at Schwarze Pumpe in Germany. 
Three sensor heads were mounted below the collector strips (the fourth one was damaged shortly before the tests). The optical signal transmission was achieved by running fibre optic cables directly from the pantograph at $15 \mathrm{kV}$ high voltage to the interrogator in the driver's cabin. To avoid creepage currents on a dirty and wet cable surface, a $20 \mathrm{~cm}$ long section of the cable was embedded in a commercially available "creepage path elongation". A video system monitored the pantograph and the catenary (Fig. 13a) during part of the test drive. From the video, a number of sudden changes in contact force were easily correlated with certain catenary elements (droppers, section insulators, switches, etc.).

This study focused on significant force changes only. However, force changes have been measured relative to a specified reference force value, which was set to the sensor system while the pantograph head was pressed to the catenary and the locomotive halted.

In Fig. 13b, an example is given where the locomotive passed a section insulator. The event occurred first on the leading collector strip. After a time delay of $50 \mathrm{~ms}$, the same event was measured by the sensors mounted below the trailing collector strip. The strips were $60 \mathrm{~cm}$ apart and the locomotive was travelling at a speed of $43 \mathrm{~km} \mathrm{~h}^{-1}$.

The OCLs run across the track in a zigzag pattern to ensure even wear on the current collector. The zigzag generates a continuous change in the position of the applied contact force on the collector strip. From the difference in the force sensor readings below one collector strip, this OCL position can be calculated. A typical example of a position plot of a righthand curve is given in Fig. 13c.

\section{Conclusions}

Two FBG sensing systems have been developed for monitoring the operational forces at the interface between current collectors and overhead contact lines of electrical railways: (i) fast force changes and impacts in the driving direction were detected by FBG sensors embedded in the collector strip; and (ii) vertical contact forces were detected by FBGbased force measuring heads below the collector strips, also comprising compensation of temperature changes as well as vertical acceleration.

The sensitivity and measurement speed of the sensor system have been proven to be sufficient for this monitoring task. However, the transducer mechanics of the force sensors and the reproducibility of the force results in dependence of dynamic force frequencies require further optimization.

\section{Data availability}

All data are stored at a database at the Leibniz-IPHT Jena (Germany), following the data policies of the institute.
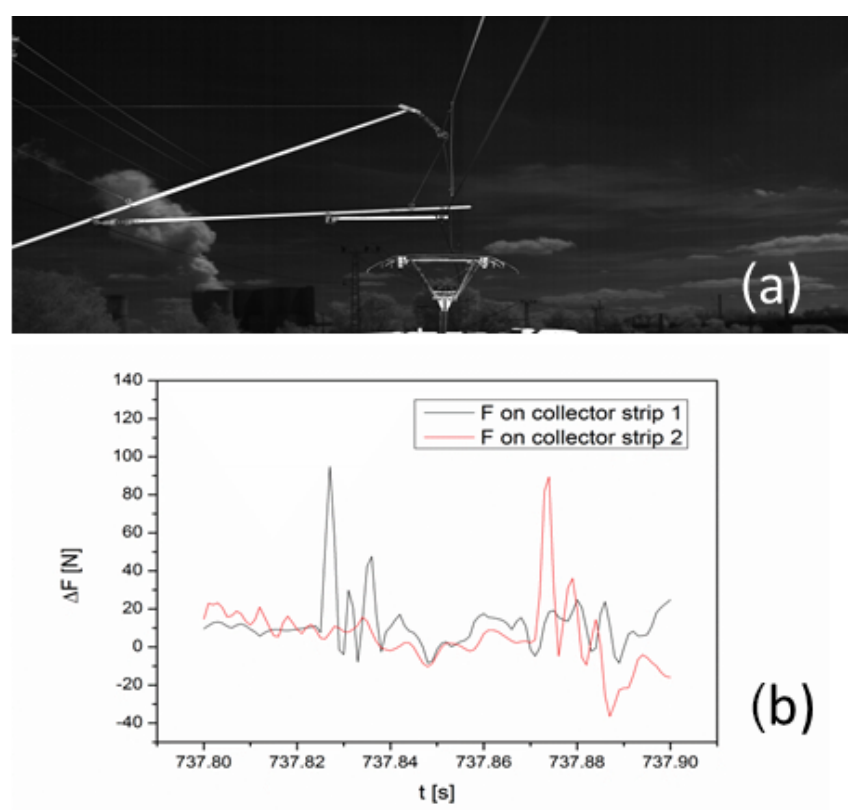

(b)

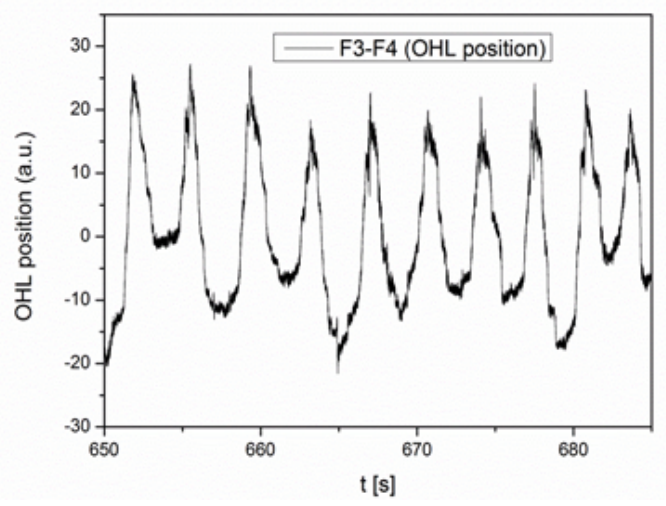

(c)

Figure 13. (a) Measurement run on tracks of the railway of the lignite-fired power plant at Schwarze Pumpe - measurement pantograph and overhead contact line with a cantilever. (b) The sum of the force sensor readings below one collector strip gives the whole force on this strip. Examining changes in these two forces can provide information on catenary anomalies, like in this example. An "event" (mass point) was measured first by the leading collector strip 1 (adapted), and after $50 \mathrm{~ms}$ it is detected by the trailing, second collector strip of the pantograph head. Both show the same signature pattern. (c) The difference in the force sensor readings below one collector strip gives the position of the overhead contact line (OHL) on the strip. Here the typical pattern of a right curve is shown.

Author contributions. Wolfgang Ecke optimized the interrogator electronics. Kerstin Schröder designed the sensors, planned and performed the measurements, and wrote the manuscript. Manfred Rothhardt helped with discussions about sensor optimization and corrected the manuscript. Uwe Richter and André Sonntag prepared and performed the tests on the locomotives and use the hit detection system regularly. Hartmut Bartelt corrected the manuscript. 
Competing interests. The authors declare that they have no conflict of interest.

Acknowledgements. This work is supported by Freistaat Thüringen (project no. TNA I-1/2012) and co-financed by the European Funds for Regional Development (EFRE), by the AiF ZIM (project no. KF2206912DB3), and by Eurailscout Inspection \& Analysis b.v. The authors thank their supporters very much.

Edited by: J. Czarske

Reviewed by: two anonymous referees

\section{References}

Antunes, P., Varum, H., and André, P.: Uniaxial fiber Bragg grating accelerometer system with temperature and cross axis insensitivity, Measurement, 44, 55-59; doi:10.1016/j.measurement.2010.09.013, 2011.

Au, H. Y., Khijwania, S. K., and Tam, H. Y.: Fiber Bragg Grating Based Accelerometer, Proc. SPIE, 7004, 70042S-1-4, doi:10.1117/12.785992, 2008.

Boffi, P., Cattaneo, G., Amoriello, L., Barberis, A., Bucca, G., Bocciolone, M., and Collina, A.: Optical fiber sensors to measure collector performance in the pantograph-catenary interaction, IEEE Sens. J., 9, 635-640, doi:10.1109/JSEN.2009.2020244, 2009.

Chojetzki, C., Rothhardt, M., Ommer, J., Unger, S., Schuster, K., and Mueller, H.-R.: High-reflectivity draw-tower fiber Bragg gratings - arrays and single gratings of type II, Opt. Eng., 44, 060101, doi:10.1117/1.1927517, 2005.
Comolli, L., Bucca, G., Bocciolone, M., and Collina, A.: First results from in line strain measurements with FBG sensors on the pantograph collector of underground trains, Proc. SPIE, 7726, 772601, doi:10.1117/12.854395, 2010.

Jiang, Q. and Yang, M.: Simulation and experimental study of a three-axis fiber Bragg grating accelerometer based on the pull-push mechanism, Meas. Sci. Technol., 24, 1-9, doi:10.1088/0957-0233/24/11/115105, 2013.

Kießling, F., Puschmann, R., and Schmieder, A.: Contact Lines for Electric Railways, Publicis, Munich, 91-94, 2011.

Mita, A. and Yokoi, I.: Fiber Bragg grating accelerometer for buildings and civil infrastructures, Proc. SPIE, 4330, 479-486, doi:10.1117/12.434148, 2001.

Schröder, K., Ecke, W., Kautz, M., Willett, S., and Jenzer, M.: An approach to continuous on-site monitoring of contact forces in current collectors by a fiber optic sensing system, Opt. Laser Eng., 51, 172-179, doi:10.1016/j.optlaseng.2012.08.007, 2012.

Schröder, K., Ecke, W., Kautz, M., Willett, S., Unterwaditzer, H., Bosselmann, T., and Rothhardt, M.: Smart current collector fibre optic hit detection system for improved security on railway tracks, Meas. Sci. Technol., 24, 1-9, doi:10.1088/09570233/24/11/115104, 2013.

Theune, N., Bosselmann, T., Willsch, M., and Puschmann, R.: Optical monitoring of highly stressed infrastructure and rolling stock modules for railway system operation, Proc. SPIE, 5502, 536539, doi:10.1117/12.566693, 2004. 